\title{
Idiopathic Pneumatosis of Small Bowel and Bladder
}

\author{
Anupam K. Gupta ${ }^{1}$, Oscar A. Vazquez ${ }^{1}$, Miguel Lopez-Viego ${ }^{1,2}$ \\ 1. Surgery, Charles E. Schmidt College of Medicine, Florida Atlantic University, Boca Raton, USA 2. Surgery, Bethesda \\ Hospital, Boynton Beach, USA
}

Corresponding author: Oscar A. Vazquez, ovazquez2017@health.fau.edu

\begin{abstract}
An 81-year-old woman presented with multiple episodes of loose bowel movements. CT scan of the abdomen and pelvis revealed pneumatosis cystoides intestinalis and asymptomatic emphysematous cystitis. The patient had an extensive workup with no obvious identified pathology to explain diffuse pneumatosis of the small bowel and bladder. Her symptoms improved with symptomatic management, empirical antibiotics, and no surgical intervention.
\end{abstract}

Categories: Urology, Gastroenterology, General Surgery

Keywords: pci, ec, surgery, gi, urology, pneumatosis

\section{Introduction}

Pneumatosis of the intestine, otherwise known as pneumatosis cystoides intestinalis (PCI), is a rare radiological finding affecting about $0.03 \%$ of the population with causes ranging from benign to lifethreatening $[1,2]$. There is no clear etiology for the pneumatosis, although it is hypothesized that it could be secondary to mechanical, infectious, or pulmonary causes, such as chronic obstructive pulmonary disease (COPD), leading to accumulation of bowel gases within the walls of visceral organs [3]. While infectious causes usually need emergent intervention, pulmonary causes can be managed conservatively; however, there is no clear consensus on when surgery is indicated besides when there is a risk of ischemia or bowel perforation [4]. One study found that up to $27 \%$ of patients presenting with benign pneumatosis underwent avoidable surgery [5]. Emphysematous cystitis (EC) is a potentially life-threatening condition that is characterized by air within the wall of the bladder as a result of infection by gas-forming organisms, but it may not always be the case. In cases where there is no clear etiology, medical management may be the most appropriate, as the pathogenesis may be similar to PCI [6-8]. However, it is unusual to see pneumatosis of the small bowel and bladder simultaneously.

Received 05/10/2020 Review began 05/18/2020 Review ended 05/18/2020 Published 05/27/2020

(c) Copyright 2020 Gupta et al. This is an open access article distributed under the terms of the Creative Commons Attribution License CC-BY 4.0., which permits unrestricted use, distribution, and reproduction in any medium, provided the original author and source are credited.

\section{Case Presentation}

An 81-year-old woman with a medical history of hypertension, hyperlipidemia, and hypothyroidism presented to the emergency room for evaluation of diarrhea for two months' duration, weakness, and fatigue. These multiple loose bowel movements were causing incontinence without hematochezia, melena, or abdominal pain. She denied associated symptoms, such as fever, symptom onset with a particular diet, or urinary symptoms. The patient had been evaluated the week prior for similar symptoms with an esophagogastroduodenoscopy (EGD), which showed evidence of moderately severe esophagitis with no bleeding in the distal esophagus, normal stomach, and normal duodenum. A colonoscopy performed the same day as the EGD was positive for large-mouthed diverticula found in the left colon, but the physical examination results were otherwise normal. Biopsies taken at that time from the esophagus, duodenum, and right and left colon revealed non-specific inflammatory changes on her pathology report. On physical examination, the patient had stable vital signs, was weak, was mildly distressed due to her symptoms, and had mild abdominal bloating. A complete blood count was unremarkable. A basic metabolic profile revealed a potassium level of $3.1 \mathrm{mmol} / \mathrm{L}$, chloride $92 \mathrm{mmol} / \mathrm{L}$, blood urea nitrogen of $27 \mathrm{mg} / \mathrm{dL}$, and a creatinine of $1.5 \mathrm{mg} / \mathrm{dL}$ from a baseline of $1.0 \mathrm{mg} / \mathrm{dL}$. Liver function tests and lipid profile were within normal limits. The patient had a mild elevation of troponins to a maximum of 0.21 , which immediately trended down on subsequent follow-up troponin tests. An electrocardiogram and echocardiogram did not reveal any abnormalities. Further workup included a CT scan of the abdomen and pelvis with intravenous contrast, which revealed diffuse pneumatosis of the small bowel (Figure 1) and bladder (Figure 2). 


\section{Cureus}

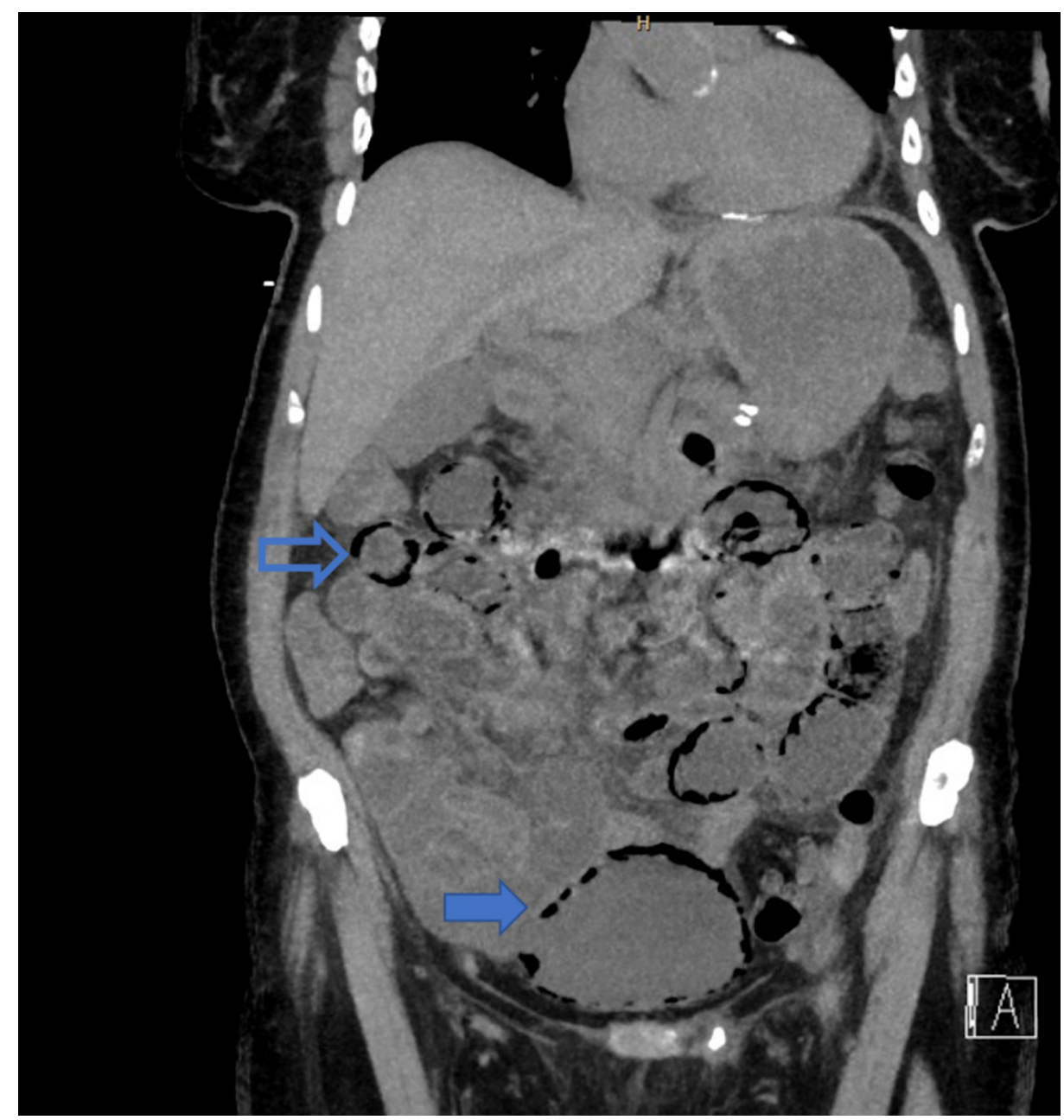

FIGURE 1: . Hollow arrow showing pneumatosis cystoides intestinalis (top) and solid arrow showing emphysematous cystitis (bottom). 


\section{Cureus}

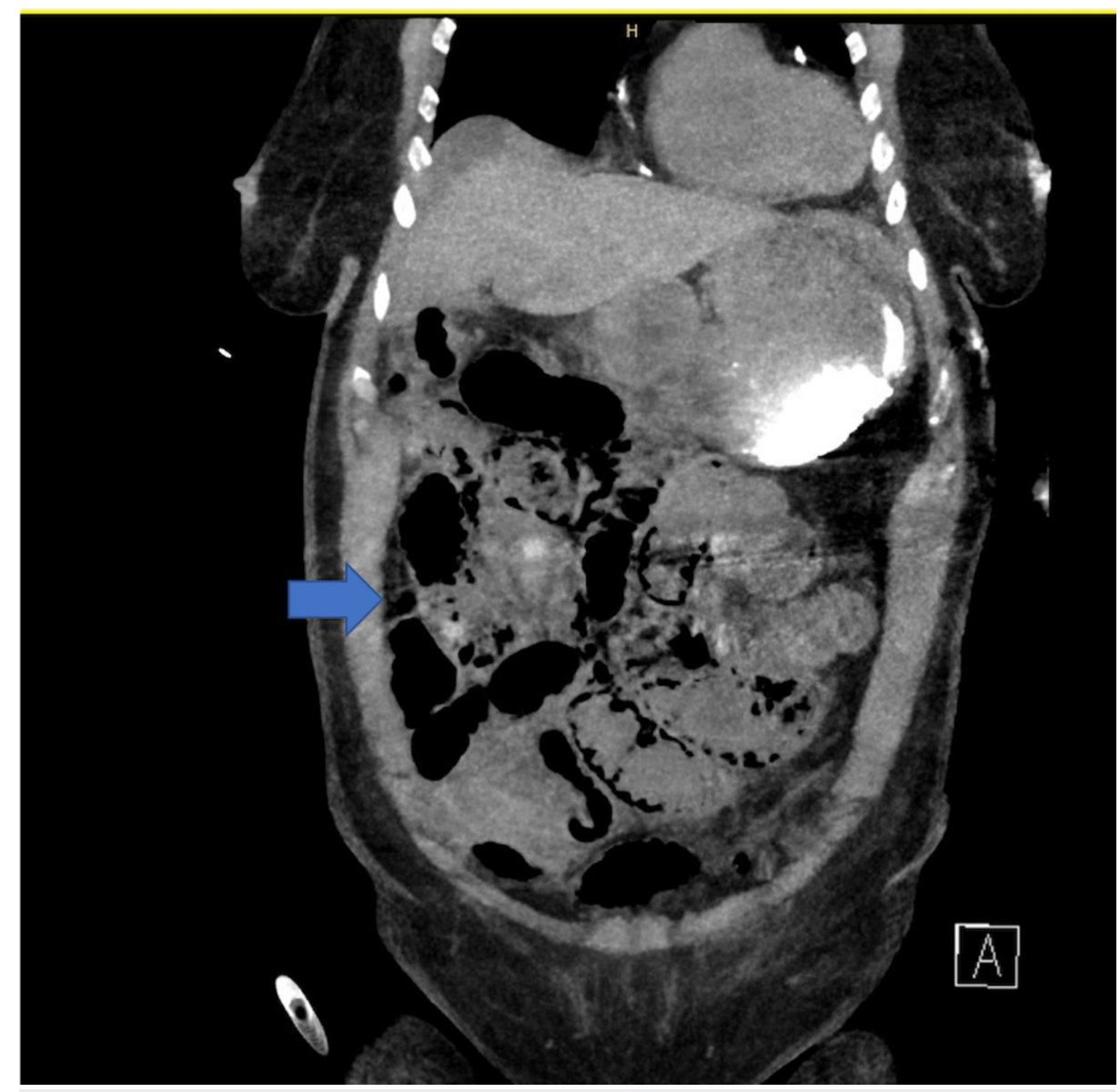

FIGURE 2: Diffuse pneumatosis of small bowel.

The patient was immediately initiated on empirical antibiotic treatment with piperacillin-tazobactam and was evaluated for a possible infectious source. This included urinalysis, stool analysis, and cultures of the urine, blood, and stool. Studies were positive for Klebsiella pneumoniae growth in the urine culture subsequently treated with a seven-day course of piperacillin-tazobactam. The patient had a Foley catheter placed to decompress the bladder with no evidence of hematuria. In light of the diffuse pneumatosis, the patient was placed on bowel rest for two days and started on a diet after that. During her entire course of seven days at the hospital, the patient was afebrile, had good urine output, and had no abdominal symptoms other than loose stools, which improved with colestipol, a bile acid sequestrant. Blood work during her stay was unremarkable except for an elevation in creatinine, which resolved with hydration, and the patient was discharged on day 7 on a regular diet (Figure 3). 


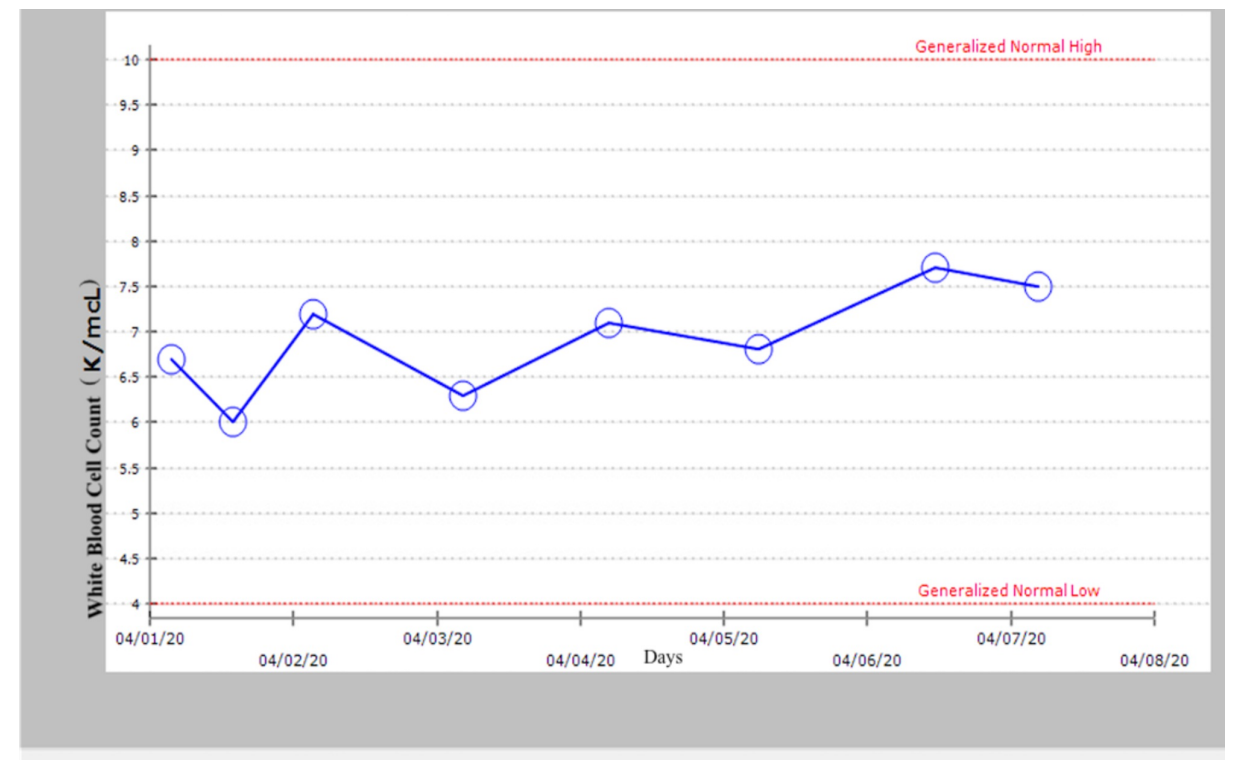

FIGURE 3: White blood count within normal limits during hospital stay.

\section{Discussion}

The exact etiology of PCI is unknown; however, it is noted that it may be related to increased intraluminal pressure caused by ileal surgery and colonoscopies, as well as other causes such as COPD, connective tissue disorders, and ingestion of sorbitol or lactulose [9]. As mentioned previously, it is theorized that the causes of PCI may be infectious, pulmonary, or mechanical. The infectious theory of PCI is based on fermenting Clostridia and Escherichia coli localizing to the submucosa and leading to the production of hydrogen gas, which is retained by the submucosa and lymphatic channels [10]. This theory is further supported by the observed resolution of pneumatosis with the use of metronidazole [11]. Pulmonary causes of PCI are theorized to be from gas freed by ruptured alveoli, which travels through the mediastinum into the perivascular spaces in the intestinal wall [12]. Mechanically speaking, it is theorized that bowel gas is pushed through a mucosal defect into lymphatic channels and spread distally by peristalsis; yet, this phenomenon does not explain hydrogen in the cysts formed $[13,14]$.

Patients with PCI do not have a characteristic presentation. They may be asymptomatic or report concerns of pain with abdominal distension, diarrhea, and hematochezia, with a mortality rate that may reach up to $75 \%$ [15]. Furthermore, patients with EC present with classic lower urinary tract infection (UTI) signs, such as urgency and dysuria, along with possible pneumaturia. It is theorized to be caused by gas-producing organisms, high glucose concentration in tissues, and impaired tissue perfusion, as is often seen in middleaged women with diabetes. Other risk factors for EC are chronic UTIs, indwelling urethral catheters, urinary tract outlet obstruction, or neurogenic bladders $[6,7]$.

Laboratory tests that may be helpful include white blood cell count, aspartate aminotransferases, alanine aminotransferases, alkaline phosphatase, $\mathrm{pH}$, bicarbonate, lactic acid, and amylase. These tests must be coupled with physical exam findings such as pain, diarrhea, fever, tenderness, rectal blood loss, and hypotension, as these can indicate increasing severity of presentation, which may require surgery [15]. For PCI, CT is more sensitive than plain radiography in distinguishing it from intraluminal air or submucosal fat since it can visualize the presence of air in the bowel wall. Additionally, CT is able to detect other more worrisome findings that may suggest an underlying cause of PCI (bowel wall thickening, altered contrast mucosal enhancement, dilated bowel, soft tissue stranding, portal air, and ascites) [16]. EC is also better diagnosed by CT, as it can better visualize the gas in the bladder wall. CT can also differentiate EC from vesicocolic fistula, intra-abdominal abscess, or an adjacent neoplastic malignancy [17].

Oxygen therapy has been suggested as the primary treatment for asymptomatic PCI, with antibiotic treatment reserved for EC, as it is most commonly due to bacterial causes if there are signs of infection. Otherwise, it is treated as PCI, even with the presence of free intraperitoneal air [18,19]. Oxygen treatment is based on increasing the partial pressure of oxygen in the blood and thus increasing the pressure gradient of the gas in the cysts. The cysts then release gases contained within them and refill with oxygen, which is then metabolized, leading to resolution $[9,19]$. Surgery is mostly indicated for intestinal and extra-intestinal complications from PCI and EC, such as obstruction caused by cysts, perforation from stercoral ulceration, adhesions, or compression of adjacent structures by large masses of cysts [20]. The presenting patient had diarrhea, mild abdominal distention, and no urinary symptoms concerning for UTI. It was only on CT scan that there was a manifestation of both PCI and EC. Laboratory results revealed an elevated troponin level 
and an elevated creatinine level, which resolved with fluid hydration. She had a positive history for colonoscopy and EGD a week before for her diarrhea workup, which could cause PCI, but it would not explain EC. It may be possible that it was a bacterial manifestation causing EC as there was an infectious source based on the positive urine culture; however, with blood cultures and repeat urine cultures being negative, this was unlikely the cause of the pneumatosis. The patient was ultimately discharged after completing seven days with a broad-spectrum antibiotic treatment for the UTI.

\section{Conclusions}

Idiopathic, simultaneous asymptomatic pneumatosis of the small bowel and bladder is unusual and can be managed conservatively in a non-operative fashion if there are no concerns for intra- or extra-intestinal complications associated with PCI and/or EC.

\section{Additional Information \\ Disclosures}

Human subjects: Consent was obtained by all participants in this study. Conflicts of interest: In compliance with the ICMJE uniform disclosure form, all authors declare the following: Payment/services info: All authors have declared that no financial support was received from any organization for the submitted work. Financial relationships: All authors have declared that they have no financial relationships at present or within the previous three years with any organizations that might have an interest in the submitted work. Other relationships: All authors have declared that there are no other relationships or activities that could appear to have influenced the submitted work.

\section{References}

1. Slesser AA, Patel PH, Das SC, Leahy A, Livingstone J, Riaz AA: A rare case of segmental small bowel pneumatosis intestinalis: a case report. Int J Surg Case Rep. 2011, 2:185-187. 10.1016/j.ijscr.2011.06.003

2. Pneumatosis intestinalis imaging. (2013). Accessed: May 7, 2020: https://emedicine.medscape.com/article/371955-overview.

3. Dawe N, Akhtar S: Pneumatosis intestinalis presenting with a pneumoperitoneum in a patient with chronic bronchiectasis: a delayed diagnosis of superior mesenteric artery ischaemia. BMJ Case Rep. 2010, 2010:bcr0120102622. 10.1136/bcr.01.2010.2622

4. Blair HA, Baker R, Albazaz R: Pneumatosis intestinalis an increasingly common radiological finding, benign or life-threatening? A case series. BMJ Case Rep. 2015, 2015:2014207234. 10.1136/bcr-2014-207234

5. Jamart J: Pneumatosis cystoides intestinalis. A statistical study of 919 cases . Acta Hepatogastroenterol. 1979, 26:419-422.

6. Thomas AA, Lane BR, Thomas AZ, Remer EM, Campbell SC, Shoskes DA: Emphysematous cystitis: a review of 135 cases. BJU Int. 2007, 100:17-20. 10.1111/j.1464-410X.2007.06930.x

7. Nozu T: Emphysematous cystitis with air bubbles in the inferior vena cava . Int J Urol. 2008, 15:947. 10.1111/j.1442-2042.2008.02127.x

8. Medina-Polo J, Nuñez-Sobrino JA, Díaz-González R: An unusual case of air within the bladder wall: bladder pneumatosis?. Int J Urol. 2011, 18:375-377. 10.1111/j.1442-2042.2011.02748.x

9. Azzaroli F, Turco L, Ceroni L, et al.: Pneumatosis cystoides intestinalis. World J Gastroenterol. 2011, 17:4932-4936. 10.3748/wjg.v17.i44.4932

10. Gagliardi G, Thompson IW, Hershman MJ, Forbes A, Hawley PR, Talbot IC: Pneumatosis coli: a proposed pathogenesis based on study of 25 cases and review of the literature. Int J Colorectal Dis. 1996, 11:111-118. 10.1007/s003840050031

11. Tak PP, Van Duinen CM, Bun P, et al.: Pneumatosis cystoides intestinalis in intestinal pseudoobstruction. Resolution after therapy with metronidazole. Dig Dis Sci. 1992, 37:949-954. 10.1007/BF01300397

12. St. Peter SD, Abbas MA, Kelly KA: The spectrum of pneumatosis intestinalis . Arch Surg. 2003, 138:68-75. 10.1001/archsurg.138.1.68

13. Galandiuk S, Fazio VW: Pneumatosis cystoides intestinalis. A review of the literature . Dis Colon Rectum. 1986, 29:358-363. 10.1007/BF02554132

14. Gillon J, Tadesse K, Logan RF, Holt S, Sircus W: Breath hydrogen in pneumatosis cystoides intestinalis . Gut. 1979, 20:1008-1011. 10.1136/gut.20.11.1008

15. Knechtle SJ, Davidoff AM, Rice RP: Pneumatosis intestinalis. Surgical management and clinical outcome . Ann Surg. 1990, 212:160-165. 10.1097/00000658-199008000-00008

16. Olson DE, Kim YW, Ying J, Donnelly LF: CT predictors for differentiating benign and clinically worrisome pneumatosis intestinalis in children beyond the neonatal period. Radiology. 2009, 253:513-519. 10.1148/radiol.2532090168

17. Jarrett TW, Vaughan ED Jr: Accuracy of computerized tomography in the diagnosis of colovesical fistula secondary to diverticular disease. J Urol. 1995, 153:44-46. 10.1097/00005392-199501000-00018

18. Rennenberg RJ, Koek GH, Van Hootegem P, Stockbrügger RW: Pneumatosis cystoides intestinalis, four cases of a rare disease. Neth J Med. 2002, 60:22-25.

19. Hwang J, Reddy VS, Sharp KW: Pneumatosis cystoides intestinalis with free intraperitoneal air: a case report. Am Surg. 2003, 69:346-349.

20. Goel A, Tiwari B, Kujur S, Ganguly PK: Pneumatosis cystoides intestinalis. Surgery. 2005, 137:659-660. 10.1016/j.surg.2003.12.014 